
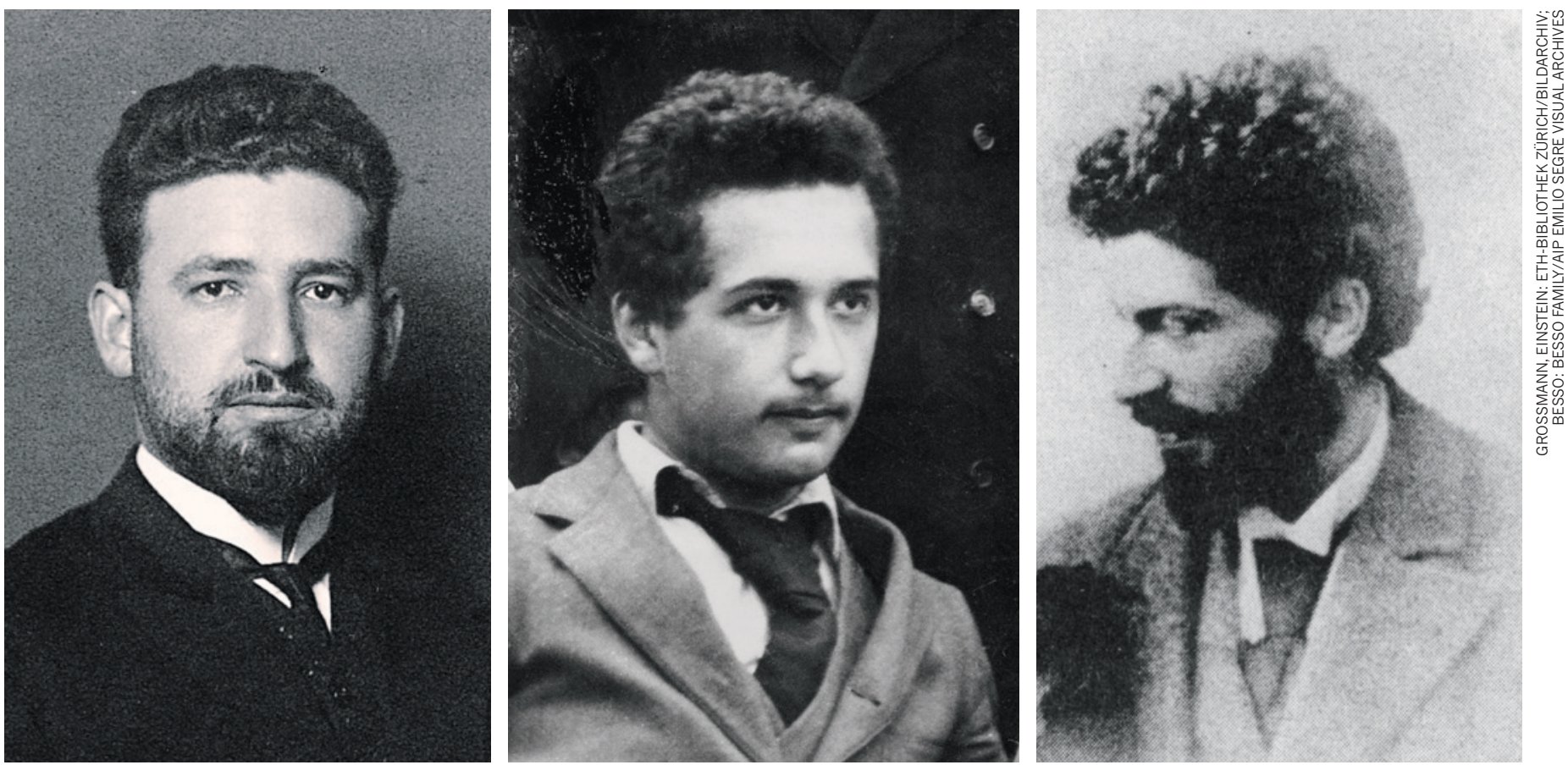

Marcel Grossmann (left) and Michele Besso (right), university friends of Albert Einstein (centre), both made important contributions to general relativity.

\title{
Einstein was no lone genius
}

\section{Lesser-known and junior colleagues helped the great physicist to piece together his general theory of relativity, explain Michel Janssen and Jürgen Renn.}

A century ago, in November 1915, Albert Einstein published his general theory of relativity in four short papers in the proceedings of the Prussian Academy of Sciences in Berlin ${ }^{1}$. The landmark theory is often presented as the work of a lone genius. In fact, the physicist received a great deal of help from friends and colleagues, most of whom never rose to prominence and have been forgotten ${ }^{2-5}$. (For full reference details of all Einstein texts mentioned in this piece, see Supplementary Information; go.nature.com/ufcgp9.)

Here we tell the story of how their insights were woven into the final version of the theory. Two friends from Einstein's student days - Marcel Grossmann and Michele Besso - were particularly important. Grossmann was a gifted mathematician and organized student who helped the more visionary and fanciful Einstein at crucial moments. Besso was an engineer, imaginative and somewhat disorganized, and a caring and lifelong friend to Einstein. A cast of others contributed too.

Einstein met Grossmann and Besso at the Swiss Federal Polytechnical School in Zurich $^{6}$ - later renamed the Swiss Federal Institute of Technology (Eidgenössische
Technische Hochschule; ETH) - where, between 1896 and 1900, he studied to become a school teacher in physics and mathematics. Einstein also met his future wife at the ETH, classmate Mileva Marić. Legend has it that Einstein often skipped class and relied on Grossmann's notes to pass exams.

Grossmann's father helped Einstein to secure a position at the patent office in Berne in 1902, where Besso joined him two years later. Discussions between Besso and Einstein earned the former the sole acknowledgment in the most famous of Einstein's 1905 papers, the one introducing the special theory of relativity. As well as publishing the papers that made 1905 his annus mirabilis, Einstein completed his dissertation that year to earn a PhD in physics from the University of Zurich.

In 1907, while still at the patent office, he started to think about extending the principle of relativity from uniform to arbitrary motion through a new theory of gravity. Presciently, Einstein wrote to his friend Conrad Habicht - whom he knew from a reading group in Berne mockingly called the Olympia Academy by its three members saying that he hoped that this new theory would account for a discrepancy of about 43 " (seconds of arc) per century between Newtonian predictions and observations of the motion of Mercury's perihelion, the point of its orbit closest to the Sun.

Einstein started to work in earnest on this new theory only after he left the patent office in 1909, to take up professorships first at the University of Zurich and two years later at the Charles University in Prague. He realized that gravity must be incorporated into the structure of space-time, such that a particle subject to no other force would follow the straightest possible trajectory through a curved space-time.

In 1912, Einstein returned to Zurich and was reunited with Grossmann at the ETH. The pair joined forces to generate a fully fledged theory. The relevant mathematics was Gauss's theory of curved surfaces, which Einstein probably learned from Grossmann's notes. As we know from recollected conversations, Einstein told Grossmann": "You must help me, or else I'll go crazy."

Their collaboration, recorded in Einstein's 'Zurich notebook', resulted in a joint paper published in June 1913, known as the Entwurf ('outline') paper. The main advance between this 1913 Entwurf 
theory and the general relativity theory of November 1915 are the field equations, which determine how matter curves space-time. The final field equations are 'generally covariant': they retain their form no matter what system of coordinates is chosen to express them. The covariance of the Entwurf field equations, by contrast, was severely limited.

\section{TWO THEORIES}

In May 1913, as he and Grossmann put the finishing touches to their Entwurf paper, Einstein was asked to lecture at the annual meeting of the Society of German Natural Scientists and Physicians to be held that September in Vienna, an invitation that reflects the high esteem in which the 34-year-old was held by his peers.

In July 1913, Max Planck and Walther Nernst, two leading physicists from Berlin, came to Zurich to offer Einstein a well-paid and teaching-free position at the Prussian Academy of Sciences in Berlin, which he swiftly accepted and took up in March 1914. Gravity was not a pressing problem for Planck and Nernst; they were mainly interested in what Einstein could do for quantum physics.

Several new theories had been proposed in which gravity, like electromagnetism, was represented by a field in the flat space-time of special relativity. A particularly promising one came from the young Finnish physicist Gunnar Nordström. In his Vienna lecture, Einstein compared his own Entwurf theory to Nordström's theory. Einstein worked on both theories between May and late August 1913, when he submitted the text of his lecture for publication in the proceedings of the 1913 Vienna meeting.

In the summer of 1913, Nordström visited Einstein in Zurich. Einstein convinced him that the source of the gravitational field in both their theories should be constructed out of the 'energy-momentum tensor': in pre-relativistic theories, the density and the flow of energy and momentum were represented by separate quantities; in relativity theory, they are combined into one quantity with ten different components.

This energy-momentum tensor made its first appearance in 1907-8 in the specialrelativistic reformulation of the theory of electrodynamics of James Clerk Maxwell and Hendrik Antoon Lorentz by Hermann Minkowski. It soon became clear that an energy-momentum tensor could be defined for physical systems other than electromagnetic fields. The tensor took centre stage in the new relativistic mechanics presented in the first textbook on special relativity, Das Relativitätsprinzip, written by Max Laue in 1911. In 1912, a young Viennese physicist, Friedrich Kottler, generalized Laue's formalism from flat to curved space-time. Einstein and Grossmann relied on this generalization

in their formulation of the Entwurf theory. During his Vienna lecture, Einstein called for Kottler to stand up and be recognized for this work ${ }^{8}$.

Einstein also worked with Besso that summer to investigate whether the Entwurf theory could account for the missing 43" per century for Mercury's perihelion. Unfortunately, they found that it could only explain 18 ". Nordström's theory, Besso checked later, gave $7 "$ in the wrong direction. These calculations are preserved in the 'Einstein-Besso manuscript' of 1913.

Besso contributed significantly to the calculations and raised interesting questions. He wondered, for instance, whether the Entwurf field equations have an unambiguous solution that uniquely determines the gravitational field of the Sun. Historical analysis of extant manuscripts suggests that this query gave Einstein the idea for an argument that reconciled him with the restricted covariance of the Entwurf equations. This 'hole argument' seemed to show that generally covariant field equations cannot uniquely determine the gravitational field and are therefore inadmissible?

Einstein and Besso also checked whether the Entwurf equations hold in a rotating coordinate system. In that case the inertial forces of rotation, such as the centrifugal force we experience on a merry-go-round, can be interpreted as gravitational forces. The theory seemed to pass this test. In August 1913, however, Besso warned him that it did not. Einstein did not heed the warning, which would come back to haunt him.

In his lecture in Vienna in September 1913, Einstein concluded his comparison of the two theories with a call for experiment to decide. The Entwurf theory predicts that gravity bends light, whereas Nordström's does not. It would take another five years to find out. Erwin Finlay Freundlich, a junior astronomer in Berlin with whom Einstein had been in touch since

"Worried that Hilbert might beat him to the punch, Einstein rushed new equations into print." his days in Prague, travelled to Crimea for the solar eclipse of August 1914 to determine whether gravity bends light but was interned by the Russians just as the First World War broke out. Finally, in 1919, English astronomer Arthur Eddington confirmed Einstein's prediction of light bending by observing the deflection of distant stars seen close to the Sun's edge during another eclipse, making Einstein a household name ${ }^{10}$.

Back in Zurich, after the Vienna lecture, Einstein teamed up with another young physicist, Adriaan Fokker, a student of Lorentz, to reformulate the Nordström theory using the same kind of mathematics that he and Grossmann had used to formulate the Entwurf theory. Einstein and Fokker showed that in both theories the gravitational field can be incorporated into the structure of a curved space-time. This work also gave Einstein a clearer picture of the structure of the Entwurf theory, which helped him and Grossmann in a second joint paper on the theory. By the time it was published in May 1914, Einstein had left for Berlin.

\section{THE BREAKTHROUGH}

Turmoil erupted soon after the move. Einstein's marriage fell apart and Mileva moved back to Zurich with their two young sons. Albert renewed the affair he had started and broken off two years before with his cousin Elsa Löwenthal (née Einstein). The First World War began. Berlin's scientific elite showed no interest in the Entwurf theory, although renowned colleagues elsewhere did, such as Lorentz and Paul Ehrenfest in Leiden, the Netherlands. Einstein soldiered on.

By the end of 1914, his confidence had grown enough to write a long exposition of the theory. But in the summer of 1915, after a series of his lectures in Göttingen had piqued the interest of the great mathematician David Hilbert, Einstein started to have serious doubts. He discovered to his dismay that the Entwurf theory does not make rotational motion relative. Besso was right. Einstein wrote to Freundlich for help: his "mind was in a deep rut", so he hoped that the young astronomer as "a fellow human being with unspoiled brain matter" could tell him what he was doing wrong. Freundlich could not help him.

The problem, Einstein soon realized, lay with the Entwurf field equations. Worried that Hilbert might beat him to the punch, Einstein rushed new equations into print in early November 1915, modifying them the following week and again two weeks later in subsequent papers submitted to the Prussian Academy. The field equations were generally covariant at last.

In the first November paper, Einstein wrote that the theory was "a real triumph" of the mathematics of Carl Friedrich Gauss and Bernhard Riemann. He recalled in this paper that he and Grossmann had considered the same equations before, and suggested that if only they had allowed themselves to be guided by pure mathematics rather than physics, they would never have accepted equations of limited covariance in the first place.

Other passages in the first November paper, however, as well as his other papers and correspondence in 1913-15, tell a different story. It was thanks to the elaboration of the Entwurf theory, with the help of 


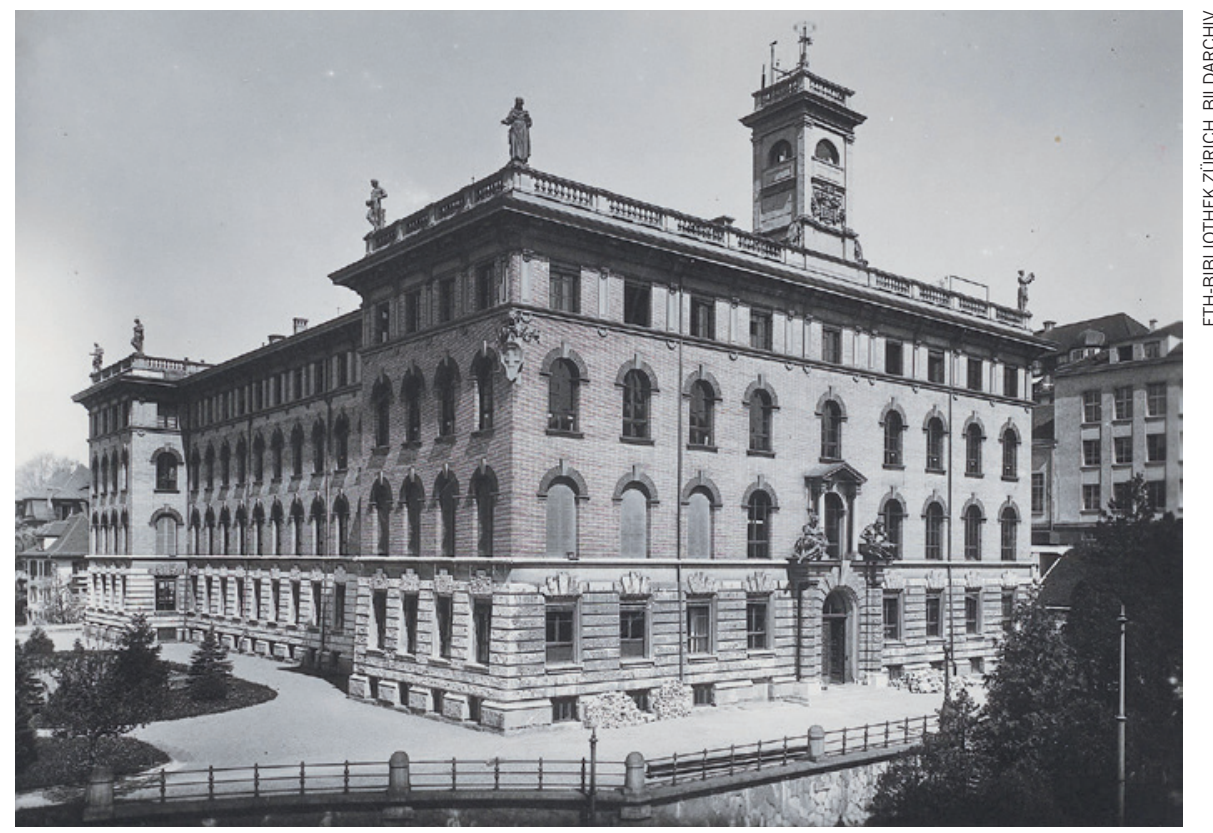

ETH Zurich, where Einstein met friends with whom he worked on general relativity.

Grossmann, Besso, Nordström and Fokker, that Einstein saw how to solve the problems with the physical interpretation of these equations that had previously defeated him.

In setting out the generally covariant field equations in the second and fourth papers, he made no mention of the hole argument. Only when Besso and Ehrenfest pressed him a few weeks after the final paper, dated 25 November, did Einstein find a way out of this bind - by realizing that only coincident events and not coordinates have physical meaning. Besso had suggested a similar escape two years earlier, which Einstein had brusquely rejected ${ }^{2}$.

In his third November paper, Einstein returned to the perihelion motion of Mercury. Inserting the astronomical data supplied by Freundlich into the formula he derived using his new theory, Einstein arrived at the result of $43^{\prime \prime}$ per century and could thus fully account for the difference between Newtonian theory and observation. "Congratulations on conquering the perihelion motion," Hilbert wrote to him on 19 November. "If I could calculate as fast as you can," he quipped, "the hydrogen atom would have to bring a note from home to be excused for not radiating."

Einstein kept quiet on why he had been able to do the calculations so fast. They were minor variations on the ones he had done with Besso in 1913. He probably enjoyed giving Hilbert a taste of his own medicine: in a letter to Ehrenfest written in May 1916, Einstein characterized Hilbert's style as "creating the impression of being superhuman by obfuscating one's methods".

Einstein emphasized that his general theory of relativity built on the work of Gauss and Riemann, giants of the mathematical world. But it also built on the work of towering figures in physics, such as Maxwell and Lorentz, and on the work of researchers of lesser stature, notably Grossmann, Besso, Freundlich, Kottler, Nordström and Fokker. As with many other major breakthroughs in the history of science, Einstein was standing on the shoulders of many scientists, not just the proverbial giants ${ }^{4}$.

Michel Janssen is professor, Program in the History of Science, Technology, and Medicine, University of Minnesota, Minneapolis, USA. Jürgen Renn is director at the Max Planck Institute for the History of Science, Berlin, Germany. e-mail:janss011@umn.edu; renn@mpiwg-berlin.mpg.de

1. Stachel, J. et al. (eds) The Collected Papers of Albert Einstein (Princeton Univ. Press, 19872015).

2. Renn, J. (ed.) The Genesis of General Relativity Vol. 2 819-830 (Springer, 2007).

3. Gutfreund, H. \& Renn, J. The Road to Relativity (Princeton Univ. Press, 2015).

4. Renn J. Auf den Schultern von Riesen und Zwergen: Einsteins unvollendete Revolution (Wiley $\mathrm{VCH}, 2006$ ).

5. Janssen, M. \& Lehner, C. (eds) The Cambridge Companion to Einstein (Cambridge Univ. Press, 2014).

6. Sauer, T. Marcel Grossmann and His Contribution to the General Theory of Relativity. Proceedings of the 13th Marcel Grossmann Meeting 456-503 (World Scientific, 2015).

7. Pais, A. 'Subtle is the Lord ... 'The Science and the Life of Albert Einstein 212 (Oxford Univ. Press, 1982).

8. Clark, R. W. Einstein: The Life and Times 156 (Knopf, 1971).

9. Norton, J. D. 'The Hole Argument' The Stanford Encyclopedia of Philosophy (ed. Zalta, E. N.) (Fall 2015 Edition); available at http://plato. stanford.edu

10. Crelinsten, J. Einstein's Jury: The Race to Test Relativity (Princeton Univ. Press, 2006). 


\section{CORRECTION}

The Comment article 'Einstein was no lone genius' (M. Janssen and J. Renn Nature 527, 298-300; 2015) wrongly stated the dates during which Albert Einstein studied at the Swiss Federal Polytechnical School in Zurich. He was there between 1896 and 1900. 